\title{
CONTRIBUIR A LA CALIDAD DEL MORIR EN EL CANCER
}

\author{
CONTRIBUTING TO THE QUALITY OF DYING DURING CANCER
}

\author{
MARITZA ESPINOZA V.* y OLIVIA SANH UEZA A.**
}

\begin{abstract}
RESUMEN
El proceso que conduce a la muerte es único e irrepetible y la forma como se viva depende de la calidad de los cuidados proporcionados. El cáncer constituye un motivo frecuente del paso a este proceso y los esfuerzos tendientes a apoyarlo se concretan a través de programas de alivio del dolor y cuidados paliativos, cuyo objetivo principal es mejorar la calidad de vida de los pacientes mientras se viva con una amenaza de muerte. La calidad de vida en estas personas se entrelaza en su significado con la calidad del morir, el que enfatizaría el enfrentar la experiencia misma de morir. Calidad del morir se puede definir cómo el grado en el cual las preferencias deuna persona para morir en el momento de su muerte concuerdan con las observaciones de como realmente murió la persona, según lo informan los demás. Esto motivó la exploración teórica y empírica de la calidad del morir en la persona con cáncer, a fin de promover el conocimiento y profundización en un tema queforma parteineludible del tipo de cuidado que deben entregar los equipos de salud que trabajan en el área.
\end{abstract}

Palabras claves: Calidad del morir, cáncer, cuidados de enfermería.

\begin{abstract}
The death process is unique and finite and the manner in which it is experienced depends on the manner and quality of care received. Cancer is often responsible for the initiation of this process. Throughout the death process, palliative care and pain control programs have the ability to enhance the quality of life of patients. The quality of the last stages of life of patients coping with Cancer is weaved within the meaning of the quality of death which leads to confronting the death experience. A person's quality of death can be defined by comparing their preferences at the end of life contrasted with the observations and information observed by others. These points motivated the theoretical and empirical exploration of the quality of death of people with Cancer, which is a necessary step to further increase the knowledge base and understanding for professionals working in this area.
\end{abstract}

Keywords: Quality of death, cancer, nursing care.

Fecha recepción: 20/12/06. Fecha aceptación: 15/03/07.

\section{INTRODUCCION}

El proceso que conduce a la muerte es vivido demaneraúnicaeirrepetible, ylaformacomo se perciba depende de la calidad del cuidado al final de la vida, constituyendo esto último un área deintervención nueva, degran importancia para las condiciones actuales de vida.
En losúltimos años hemos evidenciado los cambios demográficos en Chile, generados por la modificación en los estilos de vida y los avances en la ciencia, hacia un aumento dela morbimortalidad de enfermedades crónicas. Es así como el cáncer constituye la segunda causa demuerte, siendo una delas formas de morir más común, que implica un

\footnotetext{
* Enfermera. M agíster en Enfermería. Profesora Asistente, Depto. Enfermería, Universidad de Concepción. Concepción, Chile. E-mail: mespinoz@udec.cl

** Doctora en Enfermería. Profesora Titular, Departamento de Enfermería, Facultad de M edicina, Universidad de Concepción. Concepción, Chile. E-mail: osanhue@udec.cl
} 
proceso muchas veces prolongado en la trayectoria al final de la vida ( $M$ inisterio de Salud de Chile [M INSAL], 2002)

Debido a las implicancias del tema, tanto a nivel mundial como nacional, se hacenecesario profundizar en la forma de cuidar a las personas en el momento quesobrevieneinevitablemente la muerte y ello tiene que ver más con aspectos cualitativos que cuantitativos.

M ejorar la calidad del cuidado al final de la vida ha llegado a ser un asunto vital para pacientes, familias y seresqueridos delas personas cercanas a la muerte, al igual que para los profesionales de salud, y para quienes dictan las normas. Los esfuerzos realizados para mejorar la calidad de vida del paciente incluyen la implementación del Programa deAlivio del Dolor y Cuidados Paliativos (MINSAL, 1999), sin embargo sus logros han sido evaluados a través de medios cuantitativos, sin valorar aún la calidad de los cuidados y su repercusión en la calidad de vida y en el proceso demorir desdela perspectiva del paciente y la familia.

El presente trabajo propone describir el concepto de calidad del morir, abordando las dimensiones involucradas, los aspectos que han resultado empíricamente significativos para el paciente durante su paso hacia la muerte, y los factores que facilitarían conocer la calidad del morir.

Lo anterior supone identificar las áreas deficitarias y satisfactorias del cuidado al final de la vida, enriquecer el manejo y la relación paciente-profesional y optimizar áreas que hasta ahora han sido débilmente consideradas en su cuidado, así como también contribuir a establecer indicadores del impacto del Programa de alivio del dolor y cuidados paliativos.

\section{Prioridades para la calidad del morir en la persona con cáncer}

Cuando ya no es posible lograr la curación o controlar la enfermedad oncológica, cuando las condiciones de salud se deterioran de modo irreversibley letal, el ser humano entra en la fase terminal de su existencia. El vivir se hace progresivamente precario y penoso. Al sufrimiento físico sobrevieneel drama psicológico y espiritual del morir. Como tal, el enfermo terminal es una persona necesitada de acompañamiento humano, que la sociedad debe acoger a través de la asistencia especial del cuidado paliativo.

La transición a esta fase puedeser gradual, necesitando la actuación integrada de diversos profesionales para ayudarlo a vivir satisfactoriamentelaúltima experiencia desu vida. En esta perspectiva se intenta mejorar la calidad de vida de los pacientes a través de programas de alivio de dolor y cuidados paliativos (M INSAL,1998) y cuando la persona se enfrenta a la muerteirremediable, sehabla de calidad del morir. La calidad de vida dela persona con enfermedad oncológica avanzada tiene que ver con sus experiencias y la forma de vida mientras transita con una amenaza constante a ésta y la calidad del morir tiene quever con el tipo de experiencia dela persona al enfrentar el proceso mismo del morir (Patrick, Engelberg \& Curtis, 2001).

Según Patrick et al. (2001), calidad del morir sería una"muertequeestélibre de dolores y sufrimientos para los pacientes, familias y sus cuidadores y con los deseos del pacientey su familia".

La comprensión del concepto de calidad del morir según lo anterior implica que la persona se encuentra consciente de su muerte próxima, logrando un sentido de control para él o ella y respetando sus deseos y de sus cercanos (Patrick et al., 2001). El desconocimiento de esta situación le podría impedir vivir como protagonista de la última fase de su vida. Una persona, al estar consciente de que su vida tiene un próximo fin, quizás decida realizar actividades que nunca hizo y/o tomar decisiones importantes, como especificar su deseo a no prolongar artificialmente el proceso de su muerte.

Kübler-Ross (1969) señalaba que es nece- 
sario que los pacientes conozcan su diagnóstico y pronóstico terminal, para poder hacer frente a una situación que no es fácil. Según esta autora, serían varias las etapas por las que atraviesa una persona queconocesu diagnóstico terminal: Se iniciaría con la negación, le seguiría ira, luego depresión, pasando por una negociación, hasta llegar a la aceptación, que sería aquella etapa que le permitiría llegar en paz al final de su vida. Estas fases serían móviles y cambiantes, pudiendo ocurrir que no siempreuna persona al fallecer estéen la última etapa, la de aceptación. Esta última etapa ha sido descrita como el momento en que se despide de los suyos, arregla sus asuntos, sigue las instrucciones de los profesionales, como si ya no se tratara de él, o de su propia enfermedad o de su propio cuerpo, sino de un "ser extraño cuya morada habitaría pasajeramente". Según Gómez (1998), el paciente "razonaría de una vida", la suya, que ahora pertenece al pasado. Al mismo tiempo, espera su muerte.

Se reconoce que algunas personas moribundas desean abiertamente su advenimiento, y otras manifiestan una intensa curiosidad. Otros tienen la convicción, la alegría de encontrar del otro lado de la muerte, seres queridos y conocer el enigma de su existencia (Sason et al., 1998). La muerte para el moribundo en esta etapa significaría un umbral de percepción nueva y hasta entonces radicalmente desconocida. Cuando un enfermo hace el recorrido hasta este punto, el trabajo de los profesionales es muy gratificante y no sólo desdeel punto profesional, sino también personal y humano. Según Sureda (2005) es posible enseñar a las personas a enfrentarse con la muerte, consiguiendo que hablen de ella y la acepten como parte de la vida, pero desdeluego no constituye algo fácil de conseguir. De hecho no todos los pacientes logran llegar a este grado de aceptación.

García, García y Hernández (2001) concluyen quela mitad delos pacientesfallecen aparentemente con desconocimiento de su diagnóstico y sin saber que se enfrentan a una muerte próxima, como lo demuestra su investigación de una muestra de 70 familiares de pacientes fallecidos de cáncer: el $50 \%$ de los entrevistados opinó que el paciente no sabía que se iba a morir; el $21 \%$ que sí y el $28 \%$ lo desconocían. Esta situación de aparente desconocimiento estaba avalada por la familia, ya que son ellosque opinaban en igual porcentajequeel pacienteno debía saber, manteniendo la "conspiración del silencio", debido a un deseo de protección a su ser querido.

Franca (2005), por el contrario, señala que la mayoría de los pacientes desean saber la máxima información sobre la enfermedad. Esta posible desinformación no sólo se generaría porque el paciente o su familia no desean conocer/informarse sobre el diagnóstico y/o pronóstico, sino quetambién proviene del equipo de salud. Schaufele (1998) entrevistó un equipo desalud del Programa deAlivio del Dolor y Cuidados Paliativos en Chile, quienes respondieron en un $50 \%$ que el paciente debe conocer su diagnóstico y pronóstico; un 33\% opinó que depende delas características personales de cada individuo y un $16,7 \%$ opinó que se le debe ocultar su diagnóstico.

Como ya fue señalado, el concepto de calidad del morir involucra el conocimiento de la propia realidad para poder afrontarla, y poder decidir la forma en que se deseemorir, conforme a las preferencias personales. Estas pueden manifestarse por anticipado a través de un testamento de vida, dejando instrucciones a través de un poder o en forma verbal. El testamento puede ser un instructivo para el tratamiento, en que el paciente selecciona las terapias médicas que rechaza o que deben omitirse. Lo anterior no implica quesi el pacienteopta por ejemplo por una eutanasia voluntaria o el suicidio asistido, los profesionales deban realizarlo. El sentimiento de piedad por quien sufre un trance de agonía no justificaría la eutanasia aunque el paciente lo solicitara. "La misión no es provocar la muerte asistida, sino asistir al moribundo" (Pizzi, Muñoz \& Fuller, 2001). 
Con respecto a estas posturas extremas, en la sociedad existen diversas opiniones basadas en valores y creencias, que bajo ciertas influencias como la religión, la educación, la raza, la familia, se inclinan por apoyar una u otra. Sin embargo, en opinión de los propios pacientes, prevalecesu deseo de sobrevivir con una calidad de vida aceptable, de la cual en alguna medida somos responsables los profesionales que otorgamos el cuidado (Patrick et al., 2001).

En la actualidad la sociedad está más consciente de la necesidad dequela muerte sea en el hogar más que en un hospital. Las evaluaciones programáticas de los cuidados al final de la vida consideran exitoso que un $95 \%$ de los pacientes adscritos fallezca en su hogar (M INSAL, 1999), permitiendo así la cercanía de sus allegados significativos y supone además la ausencia de medidas de soporte vital extraordinarias, las queotorgarían más horas devida biológica, pero queno siempregarantizarían calidad en el proceso a la muerte. Por otra parte puede suceder que al fallecer en el domicilio se produzca mayor tensión en la persona y sus familiares, por desconocimiento de la forma como actuar en este trance, sobre todo si los cuidadores no están preparados o no reciben un acompañamiento cercano del equipo de salud. Por lo que el solo hecho de fallecer en el hogar no asegura por sí mismo un proceso de morir con calidad.

Dentro del contexto del hogar y al estar junto a su familia, se espera que la persona estérodeada deun entorno digno, manteniendo con los seres queridos y personas significativas contactos humanos, sencillos y enriquecedores donde se produzca la muerte, conscientes del proceso, aceptando lo quellega y como expresa Rodríguez (2002) "morir con el corazón abierto", es decir, con la despreocupación del bienestar de los que quedan con vida. Los equipos de salud pueden favorecer la comunicación deestos temastanto hacia el paciente como en los diferentes miembros de la familia o seres significativos.

La definición decalidad del morir también considera que el paciente esté lo más libre posible de dolor. El dolor, como ha sido reconocido, es una experiencia personal, individual y difícil de describir y quese ha definido como: "una experiencia sensorial y emocional desagradable, ligada a una agresión a los tejidos actual o potencial" (International Association for the study of Pain, 1979), y que contribuiría a una alta demanda de atención, ya queel $80 \%$ delos pacientescon cáncer presenta dolor en la etapa final de la vida y es uno de los aspectos que más temen las personas con cáncer avanzado. Los programas nacionales que apoyan los cuidados ponen especial énfasisen el manejo del dolor (MINSAL, 2006) y su evaluación es hecha por el propio paciente a través de la Escala Visual Análoga y en base a ésta, los profesionales indican la analgesia de acuerdo a la EscalaAnalgésica de la OMS (Organización Mundial de la Salud [OMS] 1986, citado en De Lima, 2005). Las evaluaciones del programa han considerado efectivos sus resultados, dado que al momento del egreso de éste, cerca de $94 \%$ de los pacientes manifiestan mayoritariamente dolor leve. Por otro lado, el impacto medido a través del consumo de morfina demuestra que ha aumentado considerablemente en los últimos años, desde 4 kilogramosen el año 1991 a cerca de 40 kilogramos en el año 2004 (MINSAL, 2006).

La relación paciente- profesional intervieneen la calidad del morir, ya que este último poseería los conocimientos técnicos y sería quien puede prestar el apoyo necesario para ayudar al paciente. Esta relación debeser empática, mostrando apoyo, soporte, animándolo a que hable libremente, que formule preguntasabiertas, manteniendo la capacidad de escucha (Norma de Enfermería, 1999). Existen investigaciones que señalan que la reacción que puedan presentar los pacientes crónicos ante su enfermedad va a estar en gran medida determinada por la actitud queadopte el equipo de salud (Baeza \& Peralta 1998).

Estos mismos autores han expresado que las expectativas positivas y negativas de un 
médico pueden transmitirse espontáneamente a su paciente a través de las comunicaciones verbales y no verbales.

Por otra parte, la espiritualidad (Highfield 2002) permitiría soportar el dolor y el sufrimiento, darle un sentido a la vida y generar un motor de esperanza que en muchos casos puede estar basada en la fe religiosa. A su vez autores como Herth (1989), Lazarus \& Folkman (1986), Gimenes, Quiroz y Shayer (1992) señalan que existe una relación positiva entre la esperanza y el afrontamiento de la enfermedad oncológica, más aún si se encuentra en el proceso de morir.

En la calidad del morir seintegran además cuidados que se sustentan en principios éticos, morales y humanos inherentes a la dignidad humana, la cual es compartida por toda la especie. Ellos se basan en que el ser es único, insustituible, inabarcable, irrepetible, susceptible de ser amado y capaz deamar, quees conscientey autoconsciente, goza delibertad, autodeterminación y gobierna en cierto modo al mundo y por lo mismo, es éticamente responsable. El grado de dignidad de cada ser humano no semidepor su nivel deinteligencia, por su sensibilidad ante tales o cuáles cosas o por ser más o menos libre en cada una de sus acciones 0 , por ser capaz de interrelacionarse con los otros, sino por ser uno delos individuos dela especiedetal categoría (Franca, 2005). El ser humano es digno en cada una de las etapas, desde las embrionarias a las finales, por lo que el proceso de morir debeestar revestido de máxima calidad y dignidad por ser, tal vez, la etapa más esencial de nuestra vida (Pizzi et al., 2001).

El progreso técnico-científico ha permitido un aumento de la expectativa de vida que debería por un lado ir asociado a una mejor calidad de ésta y por otro estar al servicio del ser humano, sin desvalorizar el proceso de morir, ya que de lo contrario podría generar la percepción de una vida sin significado ni sentido, haciendo que el sufrimiento pueda ser vivido como un absurdo y un destructor de la dignidad humana. A la vez, el progreso tecnológico ha favorecido el temor a la prolongación artificial de una agonía, a la pérdida de control y a la dependencia de terceros.

Por lo anterior, la calidad del morir constituye un valor ético, que no es inferior al de la curación y que conlleva el reconocimiento del valor intrínseco de la persona humana. Calidad del morir es morir con dignidad, necesitando para tal suceso, único y natural en la vida del ser humano, el respeto desus derechos como paciente terminal, enunciados universalmente por la OMS en 1990 (M INSAL, 1998).

\section{CONSIDERACIONES FINALES}

Este artículo expone la necesidad de acompañamiento quepresentan las personas en las proximidades del fin de la vida, tanto aquella que transita hacia esta etapa como los seres significativos para ella, quienes han vivido todo el proceso de duelo y que en ocasiones les es difícil enfrentar la realidad.

Todos ellos, incluso quienes han aceptado este paso natural en la vida, requieren ser acompañados por los integrantes del equipo desalud que han compartido junto a ellos esta etapa del fin de la vida.

Ellos requieren todavía orientación así como también apoyo, saber que pueden contar con al guien en aquellos momentos difíciles que se avecinan, saber que pueden Ilamarlos en horas que no son usuales, para consultar e incluso para solicitarles que vengan a estar junto a la familia.

El cáncer es una de las causales de muerte que es vivida a través de un difícil proceso y entender esto por el equipo de salud permitirá no eludir la gran responsabilidad de ayudar a las personas a morir con calidad.

Ayudar por un lado a hacer quelas personas y sus familias conozcan su realidad para poder afrontarla, para que por otro lado los miedos y ansiedades que pudieran generarse, puedan compartirlos con el equipo, les ayudará a elaborar la pérdida (Twycross, 1998). 
La cercanía del equipo de salud a través de una buena comunicación podría proporcionar los aspectos afrontativos que favorecerían una mejor calidad del morir (Lazarus \& Folkman, 1986). Para ello es necesario que el equipo de salud pueda tener las habilidades necesarias para abordar dichos temas, con lo cual se presenta la imperiosa necesidad de preparar al equipo quetrabajará en esta área.

El control de síntomas, en especial el dolor, pareciera ser uno de los aspectos prioritarios en la atención paliativa, ya quees lo que más aflige tanto al que lo sufre como a sus seres significativos. Sin embargo, también es necesario no descuidar otros síntomas, que pueden ser tanto o más aflictivos para las personas. Todo ello requieretambién una preparación específica, constante y actualizada del equipo, aunque ya posean experiencia laboral en esta área. Se reconocen además componentes éticos, espiritualesy sociales queson necesarios considerar para proporcionar una atención integral.

Enfermería como disciplina responsable de proveer el cuidado de salud y por su formación humanística, debiera entregar la asistencia necesaria a la persona y familia que atraviesa por esta etapa final de la vida. Tiene el desafío de gestionar una atención de calidad, lo cual trae consigo la necesidad deincrementar su preparación en el área y generar un mayor número deevidencia en la misma, que propenda a una mejor calidad del morir en la persona con cáncer.

\section{REFERENCIAS}

Baeza, M. y Peralta, M. (1998). Percepción dada por losfamiliares sobrecalidad deatención en relación a cuidados paliativos de enfermos oncológicosterminales. Tesis para optar al grado de Enfermera. Pontificia Universidad Católica. Chile.

De Lima, L. (2005). El uso de medicamentos opioides en el alivio del dolor por cáncer. Re cuperado el 5 de agosto de 2005 de http:// www.scare.org.co/rca/archivos/articulos/2000/
vol_3/Word/El\%20uso\%20e\%20medicamentos. doc $\bar{c}$

Franca, 0. (2005). La ayuda (médica) al buen morir. Sociedad Uruguaya deBioética. Universidad Católica deU ruguay. Recuperado el 4 de agosto 2005. de http:// escuela.med.puc.cl/ publ/ARS medica/ ARS medica 11/ARSoshtml.

García, R.; García, M.; y Fernández, I. (2001). Complicaciones Durante el Proceso deM orir. Presentado en las Jornadas de la Antigua Sociedad Española deTanatología. Las Palmas de Canaria. España. Recuperado el 10 de agosto 2004 dehttp://tanatologia.org/seit/comunicaciones.

Gimenes, M.; Queiroz, E. y Shayer, B. (1992). Reaçoes Emocionais Diante do Cancer: Sugestoes para Intervençao. Arq.Bras.M ed.; 66(4): 353. Recuperado el 6, jul-agos.2004. Dehttp:// www.unb.br/ip/labsaude/publicacoes/ elizabeth.html .

Gómez, M . (1998). Decir o no decir la verdad. (2 Ed.). Cómo dar las malas noticias en medicina (pp. 43-45). España. Ediciones Aran.

Herth, K. (1989). The relationship between level of hopeand level of coping responseand other variables in patients with cancer. Oncol Nurs Forum 16(1) 67.

Highfield, M .F. (2002). Spiritual heath of oncology patients nurseand patient perspectives. Cancer nurs. 15 (1) 1.

International Association for the study of Pain (1979). Subcommittee on Taxonomy Pain terms: a list with definition and noteson usage. Pain 3 (6): 249-252.

Kübler-Ross, E. (1969). Attitudes toward death and dying. (1 ${ }^{a}$ ed.) 25-50. On D eath and Dying. New York. Ed.M acmillan publishing Company. Recuperado 20 de agosto $2005 \mathrm{http}: / / \mathrm{www}$. amazon.com/gp/reader/0684839385/ ref $=$ sib_dp_pt/002-3957731-7599256\#treaderlink

Lazarus, R. \& Folkman, S. (1986). Estrésy procesos cognitivos (2aㅡ ed.) 124-252. Barcelona. España. Ed. M artínez Roca.

M inisterio de Salud (1998). Norma de Enfermería. Programa Nacional de Alivio del Dolor y Cuidados Paliativos. Documento oficial. Santiago. Chile.

M inisterio de Salud (1998). Programa Nacional deAlivio del Dolor y Cuidados Paliativos. Documento oficial. Santiago. Chile. 
Ministerio de Salud (1999). Cuenta anual sobre resultados del Programa Nacional deAlivio del Dolor y Cuidados Paliativos a Pacientes con Cáncer. Documento oficial. Santiago, Chile.

M inisterio deSalud deChile(2002). Departamento de estadísticas e información de salud. Indicadores de estadísticas vitales, según región, año 2002. Recuperado 2 denoviembrede2004. Disponible en http://deis.minsal.cl/Indicadores/ind2004.pdf

Ministerio de Salud (2006). Resultados estadísticos del Programa Alivio del Dolor y Cuidados Paliativos. Trabajo presentado en Congreso Latinoamericano de Cuidados paliativos. Noviembre. Santiago, Chile.

Patrick, D., Engelberg, R. \& Curtis, R. (2001, septiembre). Evaluating the Quality of Dying and Death. Journal of Pain and Symstom M anagement 22 (3): 717-726.
Pizzi, P., M uñoz, A. \& Fuller, A. (2001). El dolor, la muertey el morir ( $1^{\text {a }}$ ed.). 65-80. Santiago, Chile: Editorial M editerráneo.

Rodríguez, P. (2002). El enfermo terminal y el derecho a una muerte digna (1a ed). 169-184. M orir es nada. España: Ediciones Barcelona.

Sason, F., Girgis, A., Boyes, A., Bonevski, B., Burton, L. \& Cook, P. (1998). The unmet supportive care needs of patients with cancer. Cancer 88: 225-36.

Sureda, M. (2005). Aproximación del enfermo terminal a la muerte. Temas Bioéticos. Asociación Catalana de estudios Bioéticos. Recupe rado el 14 de marzo 2005 de http://www. Aced.org/term.htm

Twycross, R. (1998). Palliative Care. In: Encyclopedia of Applied Ethics 3(15): 419-433. 